\title{
Histoire du Bulletin \\ de la Société Française de Physique
}

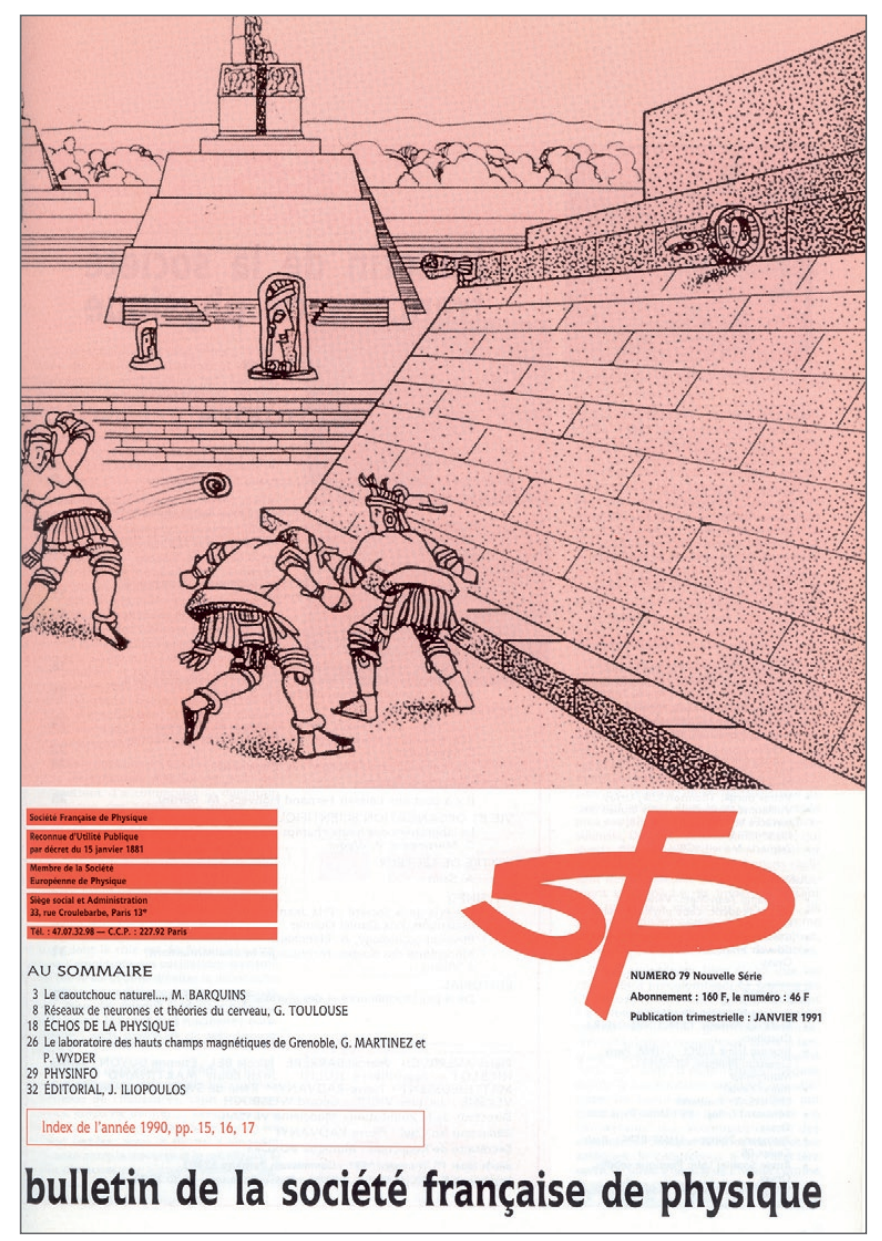

\section{Introduction}

Le Journal de Physique Théorique et Appliquée fut fondé en 1872, et la Société Française de Physique fut créée en 1873. Un autre journal, Le Radium, vit le jour en 1904. Ces deux revues scientifiques furent fondues en une seule, le Journal de Physique et le Radium, par les soins de Paul Langevin, en 1920. Cette publication, renommée par la suite simplement Journal de Physique, fut éditée jusqu'à la fin de 1997.

La Société Française de Physique organisa pendant près d'un siècle des séances de présentation de communications scientifiques, d'abord à Paris puis également dans les grands centres universitaires de province, qui donnèrent lieu jusqu'à la dernière guerre à la publication plus ou moins régulière de bulletins des Séances de la Société Française de Physique. Dans les années d'après-guerre, les résumés de ces communications furent publiés à la fin de certains numéros du Journal de Physique. Le nombre de physiciens allait alors croissant et leur spécialisation fut de plus en plus poussée. Ceci conduisit à l'organisation de colloques, nationaux et internationaux, de plus en plus nombreux. Dans ce contexte naquirent aussi nos congrès généraux.

Selon les époques, les Bulletins des Séances comportaient également des informations sur la vie et les activités de la SFP. Ces informations cessèrent en mai 1940. Un petit bulletin particulier, paraissant irrégulièrement, leur fut à nouveau consacré de juillet 1961 à juillet 1967. Ce fut le modeste précurseur des bulletins dont l'histoire est évoquée ci-après.

Charles de Novion et Pierre Radvanyi
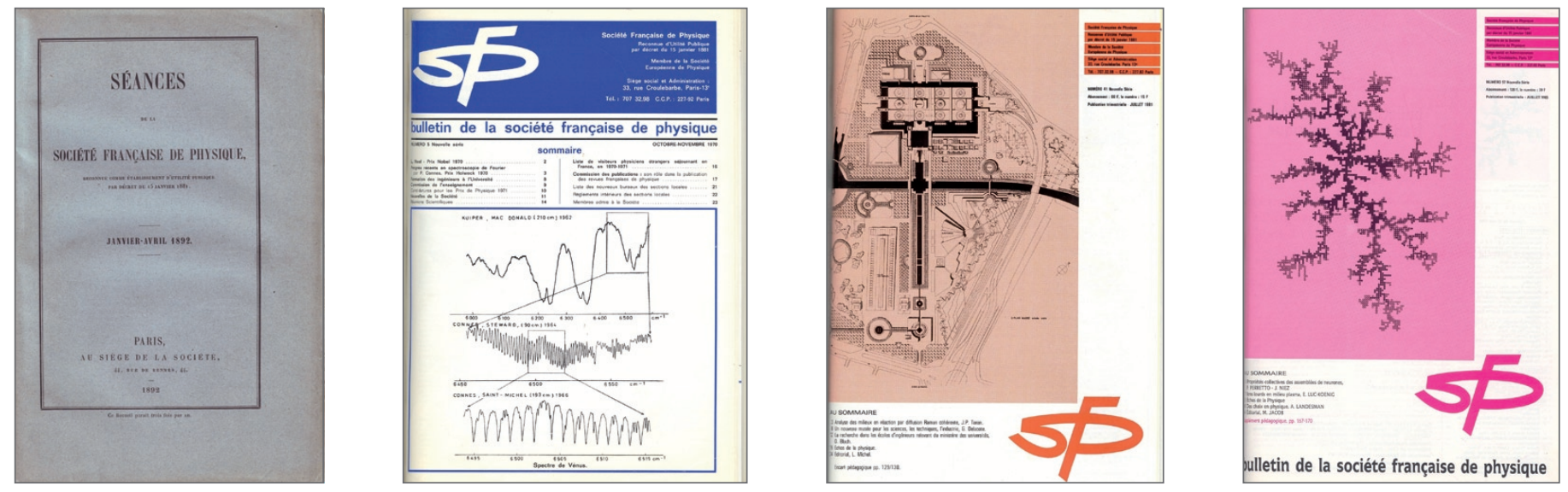

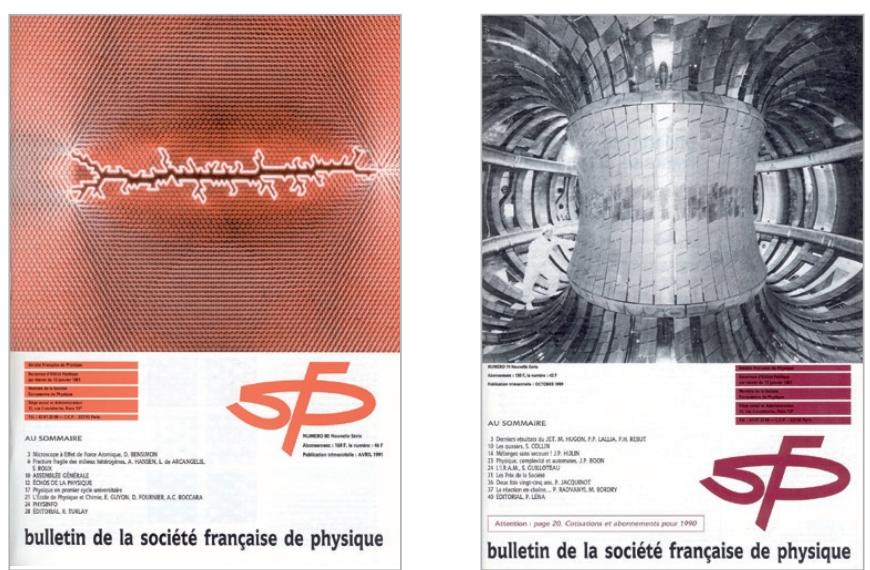

\section{En feuilletant les anciens bulletins de la SFP}

Jusque-là je n'avais organisé que des congrès en physique nucléaire et n'avais que peu d'expérience de la diversité de la Société Française de Physique, lorsque, au printemps 1969, Francis Netter, le secrétaire général d'alors, appuyé par André Herpin le précédent secrétaire général, me demanda de prendre en charge l'édition d'un nouveau Bulletin de la SFP (un « vrai » bulletin, dirent-ils, à l'instar des sociétés sœurs anglo-saxonnes). Ce travail de rédacteur en chef était tout nouveau pour moi ; je ne me doutais pas alors du nombre de bulletins (108) pour lesquels j'aurais à exercer cette tâche. Il n'existait à cette époque qu'un modeste bulletin d'information de taille réduite, à couverture vert clair. Une réunion se tint alors à Orsay à laquelle participèrent aussi André Guinier et André Berthelot, Président de la Société. Il fut décidé de publier 3 à 4 numéros par an, au format A4, en utilisant largement le "bleu SFP ». Le nouveau bulletin devait rendre compte des différentes activités de la Société et faire connaître ses principales manifestations, assemblées générales et remises des prix, en publiant les textes des conférences prononcées à ces occasions. Les éditoriaux seraient demandés aux Présidents en exercice de la SFP et le Bureau apporterait toute son aide. Il fallait également une secrétaire de rédaction : Monique Bordry, qui m'avait déjà efficacement assisté dans l'organisation d'un congrès international, accepta de prendre cette fonction. Notre premier numéro (Bulletin $\mathrm{n}^{\circ}$ 1, Nouvelle Série) parut à l'automne 1969 avec un éditorial d'André Berthelot.

Feuilletons les pages des premiers de ces bulletins. On retrouve les comptes rendus des assemblées générales avec les conférences qui les accompagnent, les prix de la Société, en particulier le prix Holweck et le prix Ricard nouvellement créé, avec les conférences des lauréats dont plusieurs se sont vus décerner quelque temps après le prix Nobel, comme D. Gabor et Cl. Cohen-Tannoudji en 1971, B.D. Josephson et G. Charpak en 1973, A. Hewish en 1974 et A. Fert en 1994 (L. Néel, A. Kastler et P.-G. de Gennes avaient reçu le prix Holweck avant 1969). Nous trouvons également les programmes et les comptes rendus des congrès généraux de la SFP. Le premier congrès général, avec ses séances plénières et ses colloques de spécialité, s'est tenu à Évian en 1971, le deuxième - celui du centenaire de la SFP - à Vittel en 1973, suivi en 1974, à l'initiative de N. Kurti, par un congrès franco-britannique à Jersey en l'honneur du centième anniversaire des deux sociétés de physique. Quelqu'un au bureau, peut-être $\mathrm{R}$. Castaing, remarqua que les villes d'eau avaient eu leur part et qu'il fallait maintenant songer aux vins, et le congrès suivant eût lieu à Dijon. La secrétaire de rédaction écrivait chaque fois des " Notes en marge " pour décrire l'atmosphère des congrès, et le Bureau fit parfois passer des messages par son intermédiaire.
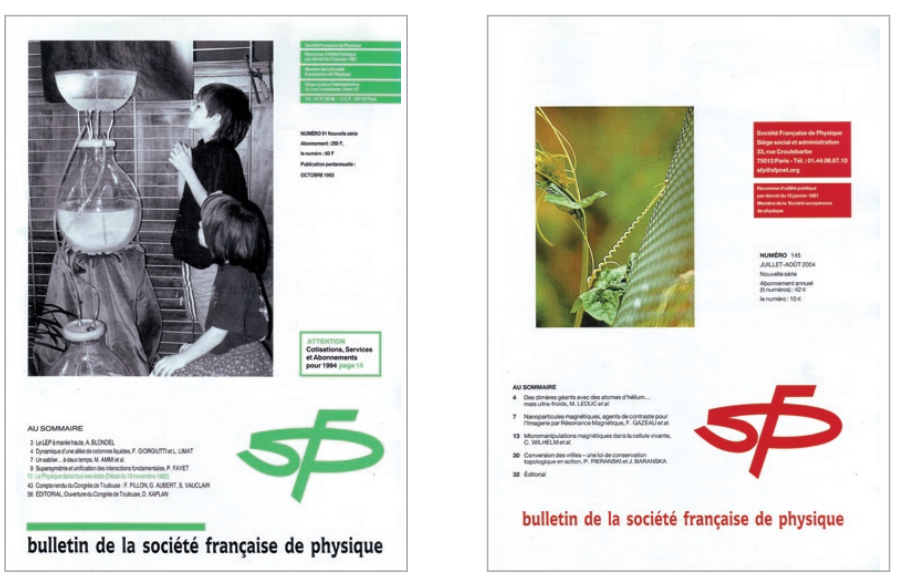

L'enseignement occupa d'emblée une place importante. La commission présidée par André Lagarrigue sur l'enseignement secondaire est créée en 1971 (Bulletin n 8) et ses travaux sont publiés dans le Bulletin. La commission SFP de l'enseignement, présidée par Michel Hulin, travaille sur l'enseignement universitaire et plusieurs pages lui sont régulièrement consacrées ; un « encart pédagogique » est publié à partir de l'automne 1972. D'autres activités de la Société sont également représentées : les débouchés, carrières et emplois des jeunes; les publications scientifiques ; la " physique dans la ville " et physique et société ; les droits de l'homme ; l'exposition de physique ; la participation à la Société Européenne de Physique. Quelques articles ont trait à l'histoire des sciences et à tel ou tel grand équipement. Une grande attention est aussi consacrée à la défense de la recherche et de ses moyens, ainsi qu'au rôle des organismes de recherche ; une conférence de Jacques Friedel (lors de la remise de la médaille d'or du CNRS), «Laisserat-on rouiller l'outil ?", reproduite dans le Bulletin, attire une large attention; il sera plusieurs fois fait appel à sa plume.

Il apparut que le Bulletin ne publiait pas assez d'informations sur la physique en train de se faire et sur les résultats obtenus. En 1979 avec le numéro 32, le Bulletin fait peau neuve : pour couvrir les différents domaines de la physique il se munit d'un comité de rédaction représentant les différentes sous-disciplines, et auquel Étienne Guyon et Pierre Averbuch notamment, chacun dans son registre, apporteront toute leur verve. Il y aura de nouvelles rubriques, "Échos de la physique " et "Vie et organisation scientifique ». Le Bulletin abandonne le "bleu SFP " et utilise d'autres couleurs. Trouver de bons auteurs pour les échos ne sera pas trop difficile, mais obtenir d'eux d'être concis et bien compréhensibles pour les collègues des disciplines voisines est beaucoup plus dur.

Le Bulletin paraît régulièrement à raison de 4 numéros par an jusqu'au début des années 90 , où il passera à 5 numéros. Nous avons au cours de toutes ces années assisté aux transformations et révolutions successives du métier d'imprimeur : de la mise en place des caractères à la main à la linotypie perfectionnée, puis à l'introduction de l'ordinateur et des disquettes, et enfin à la mise en page informatisée et à l'envoi des fichiers par internet.

Claude Sébenne reprendra le flambeau, à partir du Bulletin nº109, en 1997 ; il introduira dans chaque numéro un "billet d'humeur". Ce sera ensuite le tour de Charles de Novion qui opérera une très importante mutation : la transformation du Bulletin en Reflets de la physique avec une maquette plus esthétique et plus aérée, réalisée par de jeunes professionnelles, et dont le premier numéro est paru en septembre 2006.

Pierre Radvanyi (radva@ipno.in2p3.fr) Institut de Physique Nucléaire, Université Paris-Sud, 91406 Orsay Cedex 